\title{
Molecular association of cryptand 221D in NaCl-water solutions. A small-angle neutron scattering study
}

\author{
E. CAPONETTI, D, CHILLURA MARTINO, M.A. FLORIANO and R. TRIOLO
}

Dipartimento di Chimica Fisica, Università di Palermo, Via Archirafi 26, 90123 Palermo, Italy

\begin{abstract}
Molecules of 5-Decyl-4,7,13,16,21-pentaoxa-1,10-diaza-bicyclo-[8.8.5]tricosan (221D) and its sodium complex, with both a hydrophobic and a hydrophilic portion, are expected to form aggregates in water solutions. This was confirmed by surface tension measurements. The aggregation behaviour was studied by small-angle neutron scattering at two different $[\mathrm{NaCl}] /[221 \mathrm{D}]$ molar ratios, such as to obtain, in one case, aggregates entirely made up of ionic monomers, and in the other, mixed micelles constituted by both ionic and non-ionic units. The variation of the aggregation number and number of aggregates indicated that, in the former case, smaller micelles were formed, as a consequence of repulsion among the ionic head groups.
\end{abstract}

\section{Introduction.}

Cryptands are macrocyclic compounds having at least two basic bridgehead nitrogen atoms in the rings. Due to their cage-like structure, they can form metal complexes called cryptates [1,2] with a 1:1 metalligand ratio. Several review articles $[3,4]$ have been published on coordination and transport properties of cryptates and on cation-interaction thermodynamic and kinetic data, but, as far as we know, no studies exist on aqueous solutions of long-chain alkyl substituted cryptands.

The cryptand 5-Decyl-4,7,13,16,21-pentaoxa-1,10-diaza-bicyclo-[8.8.5]tricosan that, according to macrobicyclic ligands nomenclature, is identified as $221 \mathrm{D}$, is a derivative of the cryptand 221 obtained introducing a n-decyl hydrocarbon chain in position 5 of the tricosan ring. While 221 is very soluble in water, 221D is practically insoluble. The presence of a certain amount of salt, however, makes it soluble because of the formation of a complex between the ligand and the cation. Among the complexes formed by 221 with alkali metals, the sodium complex is the most stable [5] because the ionic radius of sodium is approximately the same as the cavity size of the cryptand. $\mathrm{A}[\mathrm{NaCl}] /[221 \mathrm{D}]$ molar ratio of 0.35 is sufficient to make the cryptand soluble in water.

Because of the high hydrofobicity of both the cryptand and the cryptate, the formation of aggregates can be expected. This hypothesis has been verified by performing surface tension, $\gamma$, measurements in water solutions at two $[\mathrm{NaCl}] /[221 \mathrm{D}]$ molar ratios, 0.4 and 1 respectively. In order to investigate the aggregation behaviour, a Small-Angle Neutron Scattering (SANS) study has been performed in $\mathrm{D}_{2} \mathrm{O}$ solutions, as a function of the cryptand 221D concentration, $\mathrm{C}$, at the above salt-cryptand molar ratios.

\section{Experimental.}

5-Decyl-4,7,13,16,21-pentaoxa-1,10-diaza-bicyclo-[8.8.5]tricosan (Kryptofix 221D, 97\% purity, from Merck) was used as received. $\mathrm{D}_{2} \mathrm{O}$ was an Aldrich product $(99.8$ atom \% D). $\mathrm{NaCl}$ was a Sigma ACS reagent. 
Surface tension measurements were performed by means of a Krüss K10 tensiometer. Solutions temperature was kept constant at $25.0 \pm 0.1^{\circ} \mathrm{C}$ by circulating fluid from an external bath.

SANS measurements were performed on the $30 \mathrm{~m}$ SANS camera of the W.C. Koheler Center for Small Angle Scattering Research at the High Flux Isotope Reactor (HFIR) in Oak Ridge USA. The samples were contained in quartz photometric cells having 0.1 and $0.2 \mathrm{~cm}$ path lengths. The temperature of the specially built cells holder was kept constant at $25.00 \pm 0.05^{\circ} \mathrm{C}$ by circulating fluid from an external bath. Neutrons of $4.75 \AA$ wavelength with sample-to-detector distances of 4.99 and 1.79 m were used. Such distances gave ranges of momentum transfer, $Q\left(Q=4 \pi(\sin \theta) / \lambda\right.$ with $2 \theta$ the scattering angle), from 0.02 to $0.12 \AA^{-1}$ and from 0.04 to $0.32 \AA^{-1}$, respectively. Scattering from samples was corrected for detector background and sensitivity, empty cell scattering, computed incoherent scattering, and sample transmission. These differences were converted to radial-averaged intensities versus $\mathrm{Q}$, and absolute elastic coherent cross sections $\mathrm{d} \Sigma(\mathrm{Q}) / \mathrm{d} \Omega$ were computed from a calibration based on the known scattering from pure water. All data corrections were performed using software provided by the Center.

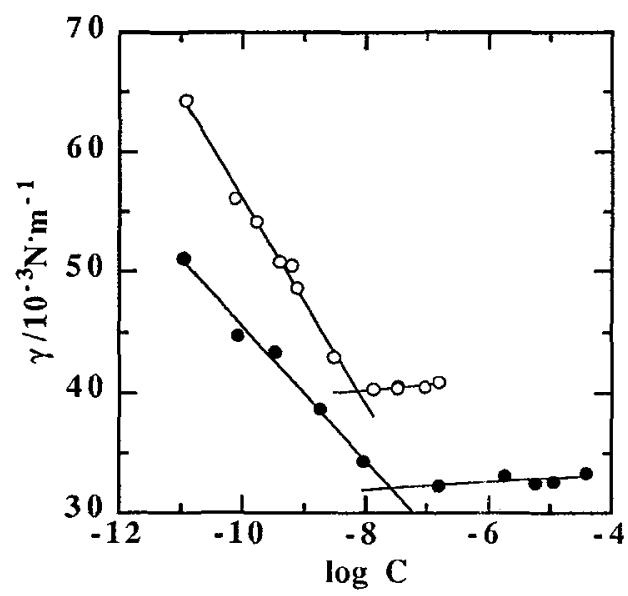

Fig. 1. - Surface tension, $\gamma$, versus $221 \mathrm{D}$ concentration at the two salt/surfactant molar ratios, $[\mathrm{NaCl}] /[221 \mathrm{D}]=0.4(\bullet)$ and 1 $(O)$, respectively. The latter points have been vertically shifted by 8 units for clarity.

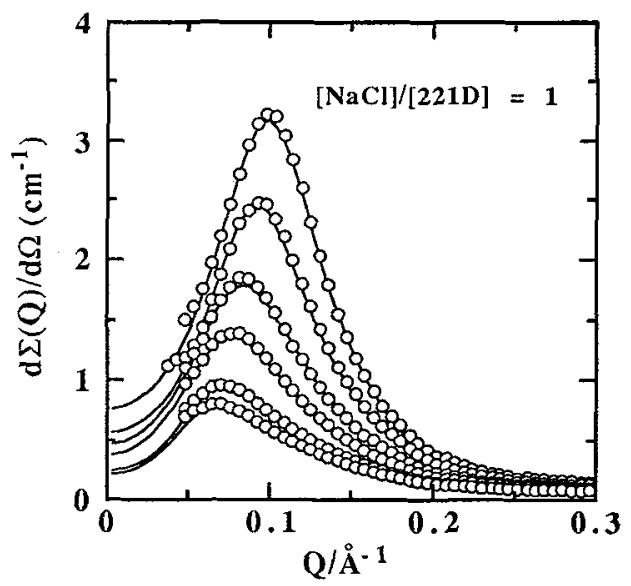

Fig. 2. - Experimental (O) and two parameter-fit (line) of SANS differential scattering cross sections at the $221 \mathrm{D}$ concentrations reported in table $\mathrm{I}$. Intensity increases with concentration.

\section{Results and Discussion.}

In figure 1 are reported plots of $\gamma$ as a function of $\log \mathrm{C}$ at the two different $[\mathrm{NaCl}] /[221 \mathrm{D}]$ molar ratios. In both cases, at a certain concentration, the surface tension plot displays a breakpoint after which $\gamma$ is practically constant. This behaviour is typical of surfactant molecules which show a sudden change in most of their solution properties at a critical micellar concentration, $\mathrm{cmc}$. This change is related to the formation of aggregates, called micelles, in which the contact between the hydrophobic portion of the molecules and water is minimized, while, at the same time, hydrophilic interaction is maximized. These aggregates are not static but, rather, highly dynamic entities, though stable enough to be detected with different techniques.

The cmc values, obtained as the intersection points of the linear fits of $\gamma$ versus $\log \mathrm{C}$ (see figure 1), were $5.0 \cdot 10^{-4}$ and $2.9 \cdot 10^{-4} \mathrm{M}$ for $[\mathrm{NaCl}] /[221 \mathrm{D}]=0.4$ and 1 respectively.

Alkyl substitutions in cryptands, generally causes an increase of the hydrophobicity and only a slight reduction in the cryptates stability [6]. Therefore it is reasonable to assume that the equilibrium constant for the sodium ion complexation by cryptand $221 \mathrm{D}$ will not be substantially different from that of cryptand 221 $\left(\log \mathrm{K}_{\mathrm{S}}=5.4\right.$ [5]). As a consequence, in what follows, it is implied that all of the sodium is present as $221 \mathrm{DNa}^{+}$. When $[\mathrm{NaCl}] /[221 \mathrm{D}]=1$, aggregates are constituted entirely by cationic surfactant monomers; when $[\mathrm{NaCl}] /[221 \mathrm{D}]=0.4$ in solution are present both the ionic surfactant $221 \mathrm{DNa}^{+}$and the nonionic surfactant 221D and, hence, mixed micelles are formed. 
Experimental SANS data at different $221 \mathrm{D}$ concentrations, for $[\mathrm{NaCl}] /[221 \mathrm{D}]=1$, are reported in figure 2. Those corresponding to $[\mathrm{NaCl}] /[221 \mathrm{D}]=0.4$ followed similar trends: in both cases the intensity increased with increasing 221D concentration. This might be caused by either an increase in aggregates number or dimension, or both.

If one makes simple assumptions on the internal structure and shape of the aggregates, and on the nature of the interaction potential, it has been shown $[7,8]$ that, for this kind of systems, the experimental SANS curve can be calculated by the following expression

$$
\mathrm{d} \Sigma(\mathrm{Q}) / \mathrm{d} \Omega=\mathrm{Np} P(\mathrm{Q}) \mathrm{S}(\mathrm{Q})
$$

where $\mathrm{Np}$ is the particle number density (in units of $\mathrm{N}_{\mathrm{A}} \cdot l^{-1}$, with $\mathrm{N}_{\mathrm{A}}$ the Avogadro number), $\mathrm{P}(\mathrm{Q})$ is the scattering of a single particle and $S(Q)$ is the structure function, related to interparticle interactions.

In figure 3 are shown the various terms of equation (1) contributing to the calculated scattering intensity for one of the compositions examined.

The model adopted implies that the aggregates are constituted by a core, made of the hydrocarbon chains, and a uniform thickness shell containing the polar and/or charged head groups, a fraction of counterions and hydration water. In the fit procedure, particles were allowed to deviate from a spherical symmetry while maintaining constant one of the core semiaxes, $a_{1}$, which was set equal to the length of the fully extended hydrocarbon chain $(14.15 \AA)$. Parameters derived from the fit procedure were: the aggregation number, $v$, that is the number of monomers in one micelle, and the total net charge of one micelle, $z$. The background was evaluated at high $Q$ in the Porod region of the scattering curve from the slope of $\mathrm{Q}^{4} \cdot \mathrm{I}(\mathrm{Q})$ versus $\mathrm{Q}^{4}$. Np was derived from the 221D concentration, cmc and $v$; the length of the two remaining core semiaxes, $a_{2}$, and the axial ratio, $\varepsilon$, were derived from $v$ and the volume of the hydrocarbon chain $\left(296.4 \AA^{3}\right)$.

In table I are reported the above parameters for all the compositions examined. Smaller aggregates are formed at the higher salt-surfactant molar ratio due to the repulsions among the charged heads; these repulsions are weaker at the lower salt-surfactant ratio because of the presence in the micelle of neutral molecules and, thus, bigger aggregates can form. It is also apparent from table I that smaller aggregates are oblate $(\varepsilon<1)$ elliptically shaped; larger aggregates are prolate $(\varepsilon>1)$ elliptically shaped.

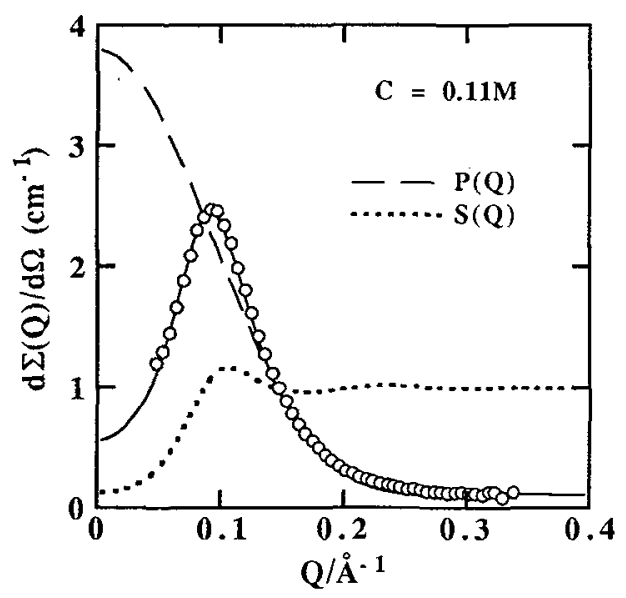

Fig. 3. - Experimental $(O)$ and calculated SANS data (solid line) for one of the sets shown in figure 2. Different terms of equation (1) are also shown.

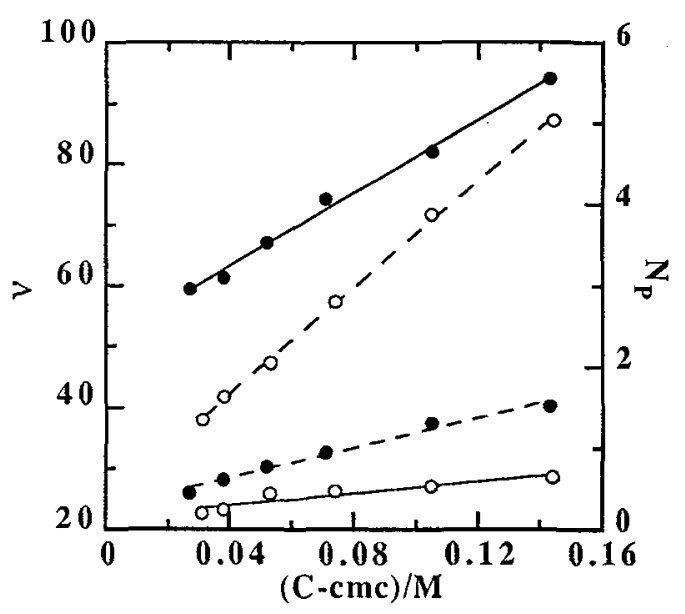

Fig. 4. - Aggregation number, $v$ (solid line), and number of particles, $\mathrm{Np}$ (dashed line), at $[\mathrm{NaCl}] /[221 \mathrm{D}]=0.4(\bullet)$ and $1(O)$, respectively. 
Table I. Results of two parameter fit of SANS data for 221D-NaCl- $\mathrm{D}_{2} \mathrm{O}$ at two different salt/surfactant molar ratios. 221D concentration, $\mathrm{C}$; aggregates number density, Np; aggregation number, $\mathrm{v}$; total net charge, $\mathrm{z}$; ellipse axial ratio, $\varepsilon$, and semiaxis, a2; thickness of the aggregates shell, Tk; background, Bk. Number in parentheses are uncertainties on the last digit.

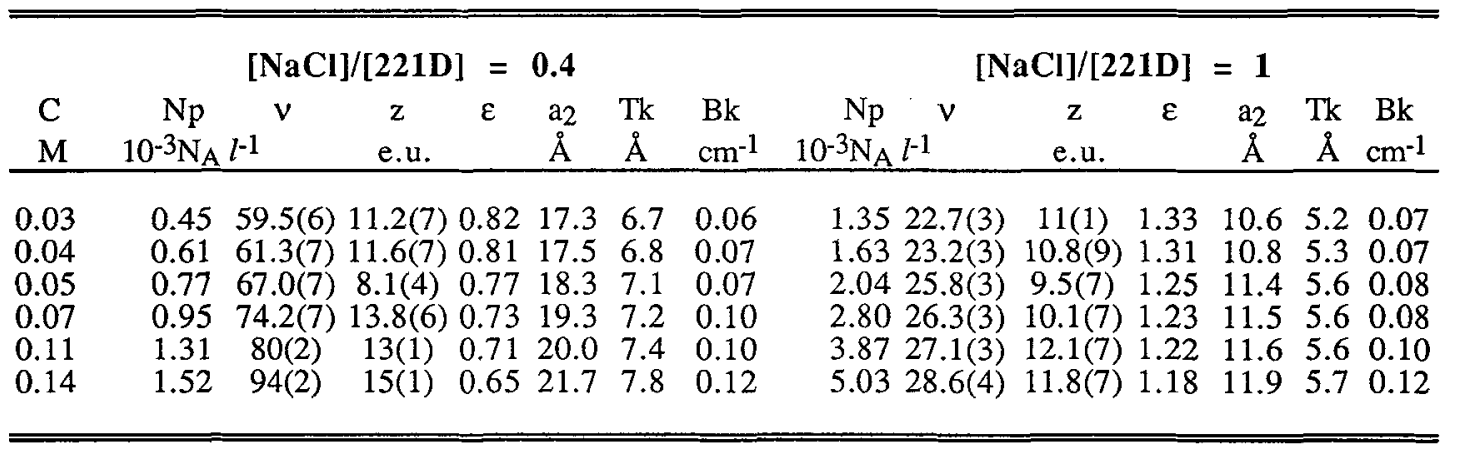

In figure 4 are reported $v$ and $\mathrm{Np}$ versus $\mathrm{C}$-cmc for the two $[\mathrm{NaCl}] /[221 \mathrm{D}]$ molar ratios. When the entirely ionic surfactant is present $([\mathrm{NaCl}] /[221 \mathrm{D}]=1)$, the slow increase in $v$ and the rapid increase in $\mathrm{Np}$ indicate formation of new aggregates on increasing the concentration. On the contrary, when mixed micelles are formed from both ionic and nonionic surfactants $([\mathrm{NaCl}] /[221 \mathrm{D}]=0.4)$, the rapid increase in $v$ and the relatively slow increase in Np indicate that growth of already present micelles is favoured. This can be rationalized in terms of repulsions among heads, as previously noted to explain the difference in aggregates size. At high salt-surfactant molar ratio, on increasing the surfactant concentration, an incoming charged monomer will be prevented from attaching itself onto an already existing micelle entirely made up of charged monomers and it is energetically more favourable to form new micelles. At the low saltsurfactant molar ratio an incoming charged or nonionic monomer will not be as strongly repelled by an already existing micelle made up of both charged and neutral monomers and the micelles can grow.

The system we have just described can be considered as an "ideal" system for studying mixed micelles, in that the only difference between the two kinds of monomers which make up the aggregates is charge. Therefore all deviations on behaviour of the mixed micelles can be univocally attributed to electrostatic interactions.

Acknowledgements. The authors are grateful to the Consiglio Nazionale delle Ricerche of Italy (CNR, Progetto Finalizzato Chimica Fine II) and to the Ministero dell' Università e della Ricerca Scientifica e Tecnologica (MURST) for financial support. They also thank the Oak Ridge W.C. Koheler Center for Small Angle Scattering Research funded by the Department of Energy.

\section{References}

[1] LEHN, J.M., Struct. Bonding (Berlin) 16 (1973) 1.

[2] LeHn, J.M. and SAUVAGE, J.P., J. Am. Chem. Soc. 97 (1975) 6700.

[3] COX, B.G. and SCHNEIDER, H., Coordination and Transport Properties of Macrocyclic compounds in Solution (Elsevier) 1992 and references therein.

[4] IZATT, R.M., BRADSHAW, J.S., NIELSEN, S.A., LAMB J.D., ChriSTENSEN J.J., and DEBABRATA S. Chem. Rev. 85 (1985) 271 and references therein.

[5] Cox, B.G., GARcia-Rosas, J. and SChneider, H., J. Am. Chem. Soc. 103 (1981) 1384.

[6] COX, B.G., FIRMAN, P., SCHNEIDER, I. and SCHNEIDER, H. Inorg. Chim. Acta 49 (1981) 153.

[7] CAPONETTI, E. and TRIOlO, R., Advances in Colloid and Interface Science, 32 (1990) 235.

[8] Caponetti, E., Floriano, M.A., VARisCo, M. and Triolo, R., Structure and dynamics of supramolecular aggregates and strongly interacting colloids, 403, S-H. Chen, J.S. Huang and P. Tartaglia ed. (Kluwer Academic Publisher) 1992. 